\title{
La politique étrangère hongroise dans le contexte de l'Europe
} centrale

Mihály Fülöp

\section{Citer ce document / Cite this document :}

Fülöp Mihály. La politique étrangère hongroise dans le contexte de l'Europe centrale. In: Politique étrangère, $n^{\circ} 1$ - 1994 59ªnnée. pp. 115-128;

doi : https://doi.org/10.3406/polit.1994.4251

https://www.persee.fr/doc/polit_0032-342x_1994_num_59_1_4251

Fichier pdf généré le 13/04/2018 


\section{Résumé}

La situation géopolitique de la Hongrie de Paprès-1989 soulève des questions relatives à sa politique de sécurité. Le gouvernement Antall a donc instauré des nouvelle bases pour la politique extérieure hongroise, parmi lesquelles figurent le rétablissement de la souveraineté totale du pays, l'élaboration d'une politique étrangère indépendante fondée sur ses intérêts nationaux, l'établissement de relations normales avec l'ensemble des Etats voisins et le rapprochement avec les pays occidentaux. Avec le projet de pacte de stabilité en Europe, la diplomatie hongroise pourra donner la priorité à deux objectifs : intégration militaire et économique à l'Ouest ; et protection des minorités hongroises, en évitant que cette question ne nuise à sa politique régionale.

\footnotetext{
Abstract

Hungarian Foreign Policy in the Context of Central Europe, by Mihály Fülöp

The geopolitical situation of post-1989 Hungary raises questions concerning its security policy. The new inaependent Hungarian foreign policy, instigated by the Antall government, is based upon national interests. It aims to fully reestablish the country's own sovereignly, establish normal relations with all neighbouring states and improve relations with Western countries. With the projected pact for European stability Hungarian diplomacy will be able to give priority to two objectives : military and economic integration with the West and the protection of Hungarian minorities without ruining its regional policy.
} 


\section{La politique étrangère hongroise Mihály FÜLöP * dans le contexte de l'Europe centrale **}

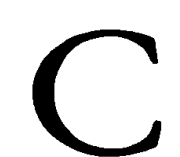

'est sans doute la Hongrie qui, aujourd'hui, jouit de la position géostratégique la plus singulière sur le continent européen. On peut aisément saisir l'essence de cette singularité en rappelant que tous les voisins de la Hongrie, sans exception, traversent une période de changements, que l'on peut qualifier de dramatiques, ce qui rend incertaines leur évolution politique interne et leurs relations extérieures.

La Hongrie est le seul pays européen qui ait des frontières avec les trois formations étatiques du continent récemment éclatées en Etats successeurs plus ou moins indépendants (Yougoslavie, Tchécoslovaquie et URSS). Mais la Hongrie a aussi un voisin, la Roumanie qui, par le biais de son union potentielle avec la Moldavie, pourrait devenir le moteur de la dernière fusion entre Etats en Europe. A l'inverse, sur sa frontière occidentale, l'Autriche tente de renforcer son ancrage "européen". Si son adhésion à l'Union européenne prend forme, l'Autriche facilitera le processus de rapprochement entamé par les pays d'Europe centrale, mais on ne peut oublier que, dans un premier temps, il faudra gérer les conséquences qu'entraîneront l'érosion du statut antérieur de l'Autriche, fondé sur sa neutralité, et la disparition des relations particulières qui associaient auparavant nos deux pays.

Le nombre des Etats frontaliers de la Hongrie est passé de cinq à sept. L'important dans cette évolution n'est pas la progression arithmétique mais l'obligation dans laquelle se trouve la Hongrie de transformer son système de relations extérieures, tant sur le plan bilatéral que régional. Ces changements ne correspondent ni à une amélioration, ni à une détérioration du cadre politique à l'échelle régionale, mais ils génèrent des phénomènes contradictoires qui compliquent nos relations extérieures.

Cet article tente de cerner les défis qui découlent de la nouvelle situation géopolitique présentée plus haut. Comment définir les nouvelles priorités de la politique extérieure hongroise? Quelle place donner à la question des minorités dans cette politique extérieure ? Comment élaborer une politique de sécurité ? Les réponses ici esquissées, à la veille des élections législatives (le

\footnotetext{
Historien, directeur de l'Institut hongrois des relations internationales (IHRI), Budapest.

Cet article a bénéficié des remarques de László Póti de l’IHRI. Par ailleurs, Paul Gradvohl (Centre interuniversitaire d'études hongroises, Paris) a bien voulu commenter une première version de ce texte. Je reste évidemment seul responsable des analyses présentées ici.
} 
premier tour se déroulera le 8 mai 1994), tiendront compte de l'évolution du consensus dans le domaine de la politique étrangère qui a longtemps associé les six formations politiques disposant actuellement d'un groupe au Parlement élu en 1990.

\section{Mise en perspective}

Tous les spécialistes de l'histoire politique de l'Europe centrale après 1945 s'accordent pour dire qu'à partir de 1964, la Hongrie a bénéficié d'une certaine latitude pour ce qui est de sa politique intérieure du fait de la "tolérance" de Moscou qui, en échange, attendait un alignement prononcé dans le domaine de la politique étrangère (cette situation se retrouve parfaitement inversée dans le cas de la Roumanie, où à la discipline la plus stricte en politique intérieure correspondait une "déviance" en politique étrangère). Cette analyse de la position de la Hongrie est pertinente pour l'essentiel, mais il faut tenir compte d'une évolution très sensible surtout quand on examine la phase finale du kadarisme et les années 1988-1990. Il ne peut y avoir de coexistence, à long terme, entre une politique intérieure et une politique extérieure qui s'ignoreraient et prendraient des voies totalement divergentes. Les tentatives répétées de réformes initiées par les acteurs de la politique intérieure hongroise aboutirent à une certaine ouverture de l'économie hongroise, qui dut se conformer de plus en plus aux normes des échanges internationaux et devint, de ce fait, plus sensible aux soubresauts de l'économie mondiale.

Ce n'est donc pas un hasard si les premières manifestations de dissension entre la politique extérieure hongroise et le cours soviétique se produisirent sur le terrain des relations économiques extérieures. L'adhésion au FMI et à la Banque mondiale, en 1982, marquèrent clairement une étape en termes de politique étrangère. Mais la dimension économique n'était pas seule en cause dans l'activité même de la diplomatie hongroise. En ce début de décennie, alors que les grandes puissances et leurs camps respectifs s'opposaient quant à l'installation des SS-20, la direction hongroise fit sentir la différence en insistant sur les intérêts et le rôle des petits Etats, et sur les dimensions non militaires de la politique de sécurité.

Une fois entrée dans l'ère postkadarienne (Kádár fut déposé en mai 1988), la diplomatie hongroise bénéficia des possibilités offertes par les changements liés au pouvoir gorbatchévien. Elle accéléra et approfondit l'abandon des fondements idéologiques (communistes) du cours précédent tout en développant une action de plus en plus fondée sur l'articulation des intérêts nationaux. La Hongrie fut la première dans le camp soviétique à établir des relations diplomatiques avec la Corée du Sud et Israël, en 1988. En 1989, la Hongrie fit une demande d'adhésion au Conseil de l'Europe, établit des relations avec l'Assemblée générale de l'OTAN et avec le Parlement européen. Cette même année - ouvrant le rideau de fer qui courait le long de sa frontière occidentale et laissant ainsi la voie ouverte à des dizaines de milliers de touristes est-allemands - la Hongrie contribua de façon significative au succès des processus de réunification européen et allemand. Les négociations sur le départ des troupes soviétiques, engagées en 1989, et qui aboutirent en mars 1990 à un accord signé par le dernier gouvernement en place avant les élections libres, montrèrent clairement et sans équivoque possible la volonté de rompre avec le camp soviétique. 
On peut finalement dire que la diplomatie hongroise a entamé sa réorientation avant même le changement de régime. Celle-ci a pris sa pleine dimension et a été mise en œuvre par la coalition arrivée au gouvernement à la suite des élections de 1990. Soulignons toutefois que, de tous les pays du camp soviétique, la Hongrie est le seul chez qui l'on puisse trouver une telle continuité en politique étrangère.

\section{Les nouvelles bases de la politique extérieure hongroise}

Le gouvernement qui s'installa en mai 1990 se fixa quelques objectifs fondamentaux en matière de politique étrangère : le rétablissement de la souveraineté totale du pays, l'élaboration d'une politique étrangère indépendante fondée sur les intérêts nationaux, la poursuite de la réorientation globale des relations extérieures du pays, de l'est vers l'ouest. Le programme du gouvernement précisait les trois grands axes des relations extérieures : la politique étrangère stricto sensu, la politique de sécurité, et les dimensions diplomatiques de l'action à l'égard des minorités [1]. Deux priorités étaient fixées concernant la politique étrangère proprement dite : prendre place dans les structures permettant l'intégration dans l'espace européen et nord-atlantique, et élargir la coopération au niveau régional en établissant des relations de bon voisinage notamment avec les anciens piliers de la Petite Entente. La politique de sécurité se devait avant tout de confirmer les bases du tournant diplomatique en cours, en assurant la rupture avec le pacte de Varsovie et, tant que celle-ci ne serait pas effective, en modifiant la nature même des activités de l'organisation cependant que, dans le cadre des relations bilatérales, la Hongrie s'assurerait du départ des troupes soviétiques dans les délais initialement fixés. Le programme gouvernemental n'indiquait aucune volonté d'adhésion à l'OTAN. La sécurité du pays devait être garantie par un réseau d'accords bilatéraux, régionaux et paneuropéens (CSCE, etc.). Le troisième volet du triptyque des relations extérieures dispose d'un statut particulier. En effet, la défense des minorités hongroises est définie à part, en tant que problème relevant d'une logique propre, et il est dit des dimensions internationales de cette question que "la responsabilité du gouvernement bongrois (y) est tout particulièrement engagée "[2].

Les priorités ainsi définies il y a bientôt quatre ans sont restées en vigueur, même si certains points évoqués plus hauts ont subi d'importantes inflexions. Dans certains cas, comme nous le verrons, on en est arrivé à des contradictions entre les priorités.

\section{La dimension euro-atlantique}

Le Conseil de l'Europe ayant accepté la demande d'adhésion déposée par la Hongrie, ce pays fut le premier des anciens membres du bloc soviétique à intégrer de plein droit le Conseil, en novembre 1990. Le nouveau gouvernement mit parallèlement l'accent sur la Communauté européenne et commença, en 1990, à négocier un accord dont la portée dépassait largement le cadre assez réduit de l'accord commercial signé auparavant. Au bout d'un an et demi de négociations, la Hongrie signa un traité d'association avec la CE (1991). Le préambule précise que la Hongrie deviendra à terme membre de l'Union européenne et que, progressivement, une zone de libre-échange sera établie avec l'Union, la disparition des barrières tarifaires et douanières étant prévue pour l'an 2000. Notons que les Douze ont prévu le maintien d'un 
protectionnisme agricole au détriment de la Hongrie tout en lui proposant, en contrepartie, des réductions asymétriques (au bénéfice de la Hongrie) des tarifs douaniers. Le processus de ratification étant achevé, l'accord est entré en vigueur le 1 er février 1994. Le gouvernement hongrois a déposé sa demande d'adhésion à l'Union européenne le 1 er avril 1994. Afin de compléter ces démarches envers l'Union européenne, la Hongrie a mené des négociations en parallèle avec l'AELE, qui ont abouti à un résultat similaire obtenu d'autant plus rapidement que l'Association n'est pas concernée par les échanges de produits agricoles.

Les institutions ayant pour vocation d'assurer la sécurité européenne étant nettement séparées de celles que nous venons de mentionner, il est logique que le rapprochement avec l'OTAN et l'Union européenne de défense ait suivi un tout autre chemin. Tout d'abord, la question du pacte de Varsovie se posait de façon beaucoup plus nette dans le contexte de la politique de sécurité. Le nouveau gouvernement hongrois prit d'ailleurs l'initiative de demander la dissolution du Pacte, ce qui permit peut-être de supprimer aussi vite cet organisme, dissous dès l'été 1991. A partir de ce moment, le gouvernement hongrois se fixa pour (nouvel) objectif d'obtenir le statut de membre de plein droit de l'Organisation du traité de l'Atlantique Nord. La diplomatie hongroise se redéploya en conséquence. Le premier ministre Antall fit une visite au siège de l'OTAN en octobre 1991. La Hongrie considère que la création du Conseil de coopération de l'Atlantique Nord (COCONA), dont elle est membre, est une étape vers le statut de membre de plein droit de l'OTAN; elle participe d'ailleurs très activement aux travaux du COCONA. En février 1994, la Hongrie a signé le document intitulé Coopération pour la paix, élaboré dans ce cadre, et la diplomatie hongroise ne cesse de renforcer son action en direction de l'OTAN.

\section{La place de la question des minorités dans la politique extérieure hongroise}

Les traités de paix signés à l'issue de la Première Guerre mondiale, qui privèrent la Hongrie historique des deux tiers de son territoire et d'environ un tiers de la population magyare, sont maintenant du ressort de l'histoire. Ils n'en sont pas moins partie intégrante du cadre de réflexion des décideurs hongrois dans le domaine de la politique étrangère, car on compte aujourd'hui quelque 3,5 millions de Hongrois dans les pays voisins, soit entre 2 et 2,5 millions en Roumanie, 600000 en Slovaquie, 400000 en Serbie, 200000 en Ukraine, et plusieurs dizaines de milliers répartis entre la Croatie, la Slovénie et l'Autriche.

Il n'est donc ni étonnant ni choquant qu'en Hongrie - la population hongroise ne représente qu'à peine trois fois celles des minorités susmentionnées - la question des minorités hongroises revête une importance particulière et soit particulièrement sensible. Toutes les forces qui comptent dans la politique hongroise s'accordent à dire que cette question mérite d'être traitée au plus haut niveau par les autorités de l'Etat. L'importance de cette question pour la diplomatie hongroise est apparue dans toute son ampleur quand le premier ministre Antall a fait sa déclaration, selon laquelle il a dit se considérer comme le premier ministre spirituel des 15 millions de Hongrois 
(soit un peu moins de 11 millions de citoyens de la République hongroise, plus de 4 millions d'âmes vivant dans les pays voisins et 1 million dans les pays occidentaux).

Pour la première fois dans la Constitution, on introduisit l'obligation pour les gouvernements hongrois de promouvoir les droits des minorités hongroises. Le texte précise que "La République hongroise se sent responsable du sort des Hongrois vivant hors de nos frontières, et elle encourage le développement des relations (de ces communautés, MF) avec la Hongrie" [3]. Quand on examine les mécanismes de prise de décision en politique extérieure, le poids de cette question se fait clairement sentir. Un organisme gouvernemental spécialisé a été créé pour mener à bien la politique à l'égard des Hongrois vivant au delà des frontières.

Au delà des principes, on a pu percevoir assez vite la volonté du gouvernement hongrois de mettre en avant cette question. Un an après son arrivée au pouvoir, Jozsef Antall déclara que les pays voisins qui ne traitaient pas bien les minorités hongroises ne pourraient compter sur des relations de bon voisinage avec la Hongrie. Dans le rapport présenté par le gouvernement, à l'occasion du deuxième anniversaire de son entrée en action, il est dit que "il n'y a pas et ne peut $y$ avoir aucune considération ou valeur qui ravalerait les minorités hongroises au rang de priorité secondaire "[4].

\section{Coopération régionale}

Les racines historiques des différentes formes de coopérations régionales connues en Europe remontent à l'époque des blocs. Pour la Hongrie, l'URSS était le pôle de référence. Aussi, la dissolution des blocs n'ayant pas pu éradiquer cet héritage, il a fallu trouver des subterfuges pour sortir des anciens ghettos. La première des techniques à être utilisée à cette fin fut la création d'organisations "subrégionales". La Communauté Alpes-Adriatique associe non pas des Etats mais des régions dont la Bavière et les provinces orientales de l'Autriche, les départements occidentaux de la Hongrie et le nord de la Slovénie.

De cette première structure est née, tout en gardant son caractère subrégional, la Pentagonale, devenue Hexagonale, avant de se transformer en Initiative centre-européenne. En 1990, l’Autriche, l'Italie, la Tchécoslovaquie, la Yougoslavie et la Hongrie lancèrent le mouvement, suivies peu après par la Pologne. Mais avec l'éclatement de la Yougoslavie, l'organisation perdait une fédération et gagnait deux Etats-membres de plein droit (la Slovénie et la Croatie) et un observateur (la Macédoine). Alors qu'à la création, le but de cette association était de prouver qu'il était possible de faire travailler ensemble des Etats-membres des deux blocs et des pays neutres, cette question ne se pose plus aujourd'hui, et l'on est passé à la réalisation de projets concrets dans des domaines où la grande politique ne pèse pas trop. Sur le plan proprement politique, il reste la volonté d'aider à la résolution des crises qui menacent ou pèsent déjà sur la région. On trouve ainsi un forum des minorités de la région au sein même de la structure de l'Initiative et les tentatives de médiation entre les multiples partenaires des conflits yougoslaves se répètent. Par sa souplesse et la modération de ses prétentions politiques, l'Iniative centre-européenne semble être l'organisation la plus à même d'inté- 
grer les pays de la région. A ce jour, l'Ukraine, la Bulgarie et la Roumanie ont manifesté leur volonté d'y participer.

D'une tout autre nature est la Coopération, instaurée dès 1989 entre la Pologne, la Tchécoslovaquie et la Hongrie, connue sous le nom des Trois (puis Quatre) de Visegrad, ville qui a accueilli le sommet de février 1991. Les trois facteurs qui expliquent sa naissance la distinguent radicalement des expériences précédemment citées. Les pays-membres ont en effet d'emblée insisté sur la communauté de sort qui les unissait d'un point de vue tant historique que culturel et géographique. De plus, les trois pays d'origine connaissaient des problèmes convergents avec l'URSS au moment du changement de régime. Enfin, tous trois ont, dès le départ, bénéficié d'un soutien particulier de l'Occident aux pays réformateurs. On constate aisément que, de ces trois éléments de départ, seul le premier reste d'actualité, si l'on peut dire, mais il ne peut suffire pour fonder une coopération régionale qui fonctionne bien. Il y a certes eu des succès, comme la réorientation vers l'Ouest des politiques étrangères et le refus commun des exigences soviétiques en matière de sécurité (1991), lesquelles auraient limité l'exercice de la souveraineté de ces pays, et comme la signature parallèle des accords d'association avec la Communauté européenne. Mais, en octobre de cette même année, les démarches communes envers l'OTAN ne donnèrent aucun résultat. En outre, comme toute la région a, depuis 1989, plié sous le vent de la réforme, l'Occident, qui avait auparavant une politique différenciée favorable à ces trois (quatre) pays, mène maintenant des actions globales en faveur de l'ensemble des pays de la région. Avec l'affaiblissement de la bipolarisation, la cohérence du groupe de Visegrad, largement fondée sur sa position intermédiaire, n'a pas manqué de s'effriter. Ce processus s'est accéléré avec l'éclatement en deux Etats du partenaire central. En effet, d'une part, la République tchèque tente de jouer cavalier seul et veut maintenant intégrer l'Europe en solitaire et, d'autre part, l'éloignement de facto des Tchèques entraîne une recomposition malaisée car les inégalités entre partenaires sont criantes (démographie, géographie - ce qui est nouveau -, et économie notamment) ce qui entraîne des tensions accrues au sein du groupe. On en est au point où la logique d'ensemble de cette construction est remise en cause.

Pourtant, les trois pays encore actifs souhaitent, à des degrés divers, maintenir ce cadre. En premier lieu, il faut citer les Polonais, qui craignent beaucoup les évolutions que leur réservent leurs voisins orientaux et qui désirent également garder l'un des symboles de leur rôle de puissance moyenne dans la région. Ensuite viennent les Slovaques, surtout intéressés par l'association avec des pays plus avancés sur la voie des mutations économiques et mieux associés aux institutions et courants d'échanges ouest-européens, ce dont ils bénéficient et comptent continuer à bénéficier. Enfin, il y a les Hongrois. Le pays n'a pas d'inquiétude majeure quant à l'attitude future de ses voisins orientaux, mais il reste une forte incertitude du côté de l'ex-Yougoslavie. De plus, la position géopolitique de la Hongrie, et notamment à cause de l'hypothèque balkanique susmentionnée, s'est terriblement dégradée par rapport à celle de Prague, entre 1989 et 1994. Le réalisme impose donc à la Hongrie de prolonger le travail déjà accompli dans le cadre de Visegrad, ce qui est d'autant plus facile qu'aucun des actuels participants ne souhaite élargir le groupe. Cette situation correspond parfaitement au désir hongrois de maintenir vivante la césure de la région en deux groupes distincts en fonction du degré de rupture avec l'ancien système. 
Pour finir, il faut évoquer l'Eurorégion carpathique qui reprend en quelque sorte le modèle "Alpes-Adriatique " en le transposant à un ensemble de régions associant la Pologne, l'Ukraine, la Slovaquie et la Roumanie à la Hongrie. Mais, dans ce dernier cas, la question des minorités interdit à la coopération de se développer de façon dynamique. Cette dernière initiative illustre pourtant la volonté hongroise d'appliquer les principes du Conseil de l'Europe en matière de politique de contacts entre régions transfrontalières, le but étant de faire progresser au niveau subrégional l'idée de relativisation du rôle des frontières.

\section{Les relations bilatérales}

Les relations de la Hongrie avec trois de ses sept voisins sont excellentes (Autriche, Slovénie et Ukraine); elles sont bonnes avec la Croatie mais posent problème, à des degrés divers, avec la Roumanie, la Slovaquie et la Serbie.

Avec l'Autriche, on a gardé du passé commun les côtés positifs, sans compter une longue expérience de relations bilatérales particulièrement fructueuses. La volonté autrichienne d'intégrer l'Union européenne est loin de peser négativement sur la Hongrie; il suffit de regarder la carte pour comprendre qu'au contraire, une Autriche membre de l'Union facilitera les relations de la Hongrie avec ses autres partenaires de l'Union. En outre, comme la question de la neutralité de l'Autriche a été réglée en accord avec Bruxelles, elle ne devrait nuire ni au rapprochement de la Hongrie avec l'Union européenne, ni à celui avec l'OTAN.

La Slovénie est, elle, un tout nouveau partenaire pour la Hongrie (la frontière commune est fort courte). Mais les relations ont tout de suite pris un aspect positif avec l'accord bilatéral sur les droits des minorités qui va bien plus loin que tout ce qui est prévu dans les accords internationaux (y compris les droits collectifs et les droits spécifiques). L'état actuel du groupe de Visegrad a fait perdre de son actualité à la demande d'adhésion de la Slovénie qui concentre maintenant son énergie en direction de Bruxelles et apparaît comme un concurrent sérieux de la Hongrie, la Pologne, la Slovaquie et la République tchèque pour la place de premier «intégré ».

Quant à l'Ukraine, c'est un peu le modèle de réussite de la diplomatie hongroise. Alors que l'URSS achevait de se décomposer et que l'Ukraine déployait tous ses efforts pour obtenir une reconnaissance sur la scène internationale, la Hongrie, de son côté, pensait à la minorité hongroise habitant la Ruthénie, c'est-à-dire la partie de l'Ukraine qui se situe à l'ouest des Carpates. En 1991, la Hongrie fut le premier pays à reconnaître l'Etat ukrainien indépendant et l'Ukraine fut le premier Etat à parapher une déclaration sur le droit des minorités nationales. Depuis, les relations politiques sont excellentes et ont été d'ailleurs renforcées par le traité hungaroukrainien qui servit de révélateur à la rupture au sein du Forum démocratique (voir plus loin "Les limites du consensus interne"). Cependant, les relations économiques restent limitées. Elles dépendent moins de la volonté des deux Etats que des conditions économiques actuelles en Ukraine.

Les relations avec la Croatie présentent un visage bien différent. D'une part, elles sont fort anciennes et, bien que du passé l'on puisse retenir des éléments contradictoires, on insiste aujourd'hui davantage sur des éléments culturels 
communs. D'autre part, la Hongrie n'a fait que suivre la Communauté européenne dans la reconnaissance de son voisin méridional, sans prendre les devants, pour des raisons évidentes. Rappelons que, si les minorités hongroises n'ont pas fait l'objet d'un accord bilatéral, c'est simplement parce que les Serbes contrôlent actuellement les territoires où elles sont traditionnellement implantées.

C'est d'ailleurs avec la Serbie que la situation a été la plus tendue. Le long de la longue frontière entre les deux Etats, l'histoire a laissé dans les mémoires bien des traces des oppositions passées et comme les combats entre la Croatie et la Serbie ont notamment eu lieu tout près de la Hongrie, il y eut de nombreuses violations de l'espace aérien hongrois de la part des Serbes, sans parler du bombardement d'un village frontalier hongrois. Les relations sont encore compliquées par le fait que la Hongrie applique les consignes d'embargo de l'ONU et de l'Union européenne, et ce, malgré les lourdes pertes financières que cela entraine pour le pays. Quand on rappelle l'existence d'une minorité de 400000 Hongrois en Serbie, on comprend que la Hongrie ait un point de vue assez modéré sur l'avenir de l'embargo et se refuse à participer aux mesures militaires de rétorsion contre les actions serbes en Bosnie.

Avec la Roumanie, les tensions ne revêtent pas les mêmes dimensions dramatiques. On connaît le lourd héritage de tensions entre Etats qui commença dès la naissance de l'Etat roumain à la fín du XIXe siècle pour ne quasiment plus cesser. Au moment de la "révolution " roumaine, fín 1989, beaucoup de Hongrois, se souvenant de 1956, ont volé au secours des "révolutionnaires" pour aider le peuple roumain à sortir de la tyrannie. La Hongrie a fourni une aide massive à la Roumanie. Gyula Horn a été le premier ministre des Affaires étrangères à se rendre à Bucarest, fin décembre 1989. Les deux gouvernements se sont accordés pour relancer leur coopération sur de nouvelles bases : la partie roumaine a promis la réouverture du consulat général de Cluj, le Front du salut national a fait une déclaration sur le droit des minorités, le 5 janvier 1990.

Malheureusement, ces excellentes intentions ne se concrétisèrent pas et, assez vite, un nouveau vent souffla. On le vit bien, lors des événements de TirguMures. Aujourd'hui, le premier résultat de l'entrée en vigueur en Roumanie des droits de l'homme les plus fondamentaux, comme les droits d'expression et de réunion, est l'affirmation la plus radicale et la plus musclée parfois des forces les plus obscures du nationalisme grand-roumain.

Au niveau du gouvernement roumain, il ne fait aucun doute que les pressions exercées par les nationalistes finissent par avoir un écho. Aujourd'hui le traité bilatéral semble loin d'être signé puisque les droits des minorités ne peuvent être évoqués. La Hongrie, tout en reconnaissant les frontières actuelles, ne désire pas inclure une clause de frontière dans le traité de base en évoquant la validité entière des traités de paix de Paris de 1947 et les accords d'Helsinki en la matière. Précisons toutefois que les bruits qui font régulièrement état des tensions hungaro-roumaines pour y voir la cause d'un prochain affrontement militaire manquent de tout fondement.

Des trois pays voisins avec lesquels il y a des problèmes, la Slovaquie est celui qui préoccupe le moins la diplomatie hongroise. Le cadre commun qu'est Visegrad permet aux deux pays de percevoir la similarité des difficultés 
auxquelles ils sont confrontés. L'éclatenent de la Fédération tchéco-slovaque a laissé en héritage aux deux nouveaux voisins le barrage de Bös/GabcikovoNagymaros et les questions posées par une importante minorité nationale dans un Etat ayant fraîchement obtenu son indépendance, et donc désireux d'affirmer son caractère national. Les questions qui se poseront quand un traité bilatéral sera envisagé, ce qui n'est pas encore le cas, ressembleront étrangement à ce qui a été vu pour les relations avec la Roumanie.

Il faut, pour finir, évoquer le voisin d'hier, devenu puissance régionale incontournable encore aujourd'hui, à savoir la Russie. L'essentiel des problèmes hérités de l'ère soviétique a été réglé. Les troupes sont parties au moment prévu, et après de longues tractations, les revendications financières des deux parties se sont effacées dans le cadre d'un renoncement mutuel à d'éventuelles compensations financières pour les dommages subis. La moitié de l'excédent commercial hongrois, qui se montait à 1,5 milliard de dollars américains, a été soldé grâce à l'acquisition de 29 MIG 29. Les négociations se poursuivent pour la moitié restante.

Il reste toutefois un problème politique, d'une portée symbolique importante : la question de l'invasion soviétique de 1956 empoisonne encore les relations bilatérales. Jusqu'au putsch de 1991, Mikhaïl Gorbatchev a refusé de condamner cette action, voire de s'excuser. Ce n'est qu'après le putsch que le ministre Andreï Kozirev, lors de sa visite à Budapest, a fait une déclaration condamnant l'intervention. Mais ce qui suit éclairera utilement le lecteur sur les sirènes qui règnent encore sur les esprits à Moscou. En effet, il était prévu que la déclaration d'Andrei Kozirev serait annexée au nouveau traité de base bilatéral signé en novembre 1992. Or, le Parlement en place jusqu'aux élections de décembre 1993 s'appliqua à repousser la ratification du traité et la prise en considération de la déclaration comme annexe du susdit traité.

\section{La politique de sécurité : une dimension difficile à maîtriser dans un contexte mouvant et incertain}

Dans la période qui a précédé le tournant, l'idée qui dominait dans les milieux d'opposition était la neutralité. D'une part, on pouvait se référer à la politique d'Imre Nagy en 1956 et, d'autre part, l'avenir de l'URSS et du pacte de Varsovie était encore des plus imprévisibles. Avec la disparition de cette problématique, les responsables hongrois durent repenser entièrement la politique de sécurité du pays. Dans un premier temps, il y eut une tentative de mise au point d'une politique de défense tous azimuts mais, très vite, les voisins réagirent $\mathrm{mal}$ et il fallut repartir de zéro.

C'est en 1991 qu'on arriva à formuler une nouvelle conception qui reçut l'assentiment du Parlement après avoir été mise au point par les ministères de la Défense et des Affaires étrangères. On avait fini par prendre en compte les phénomènes d'éclatement en cours dans les anciennes fédérations voisines. Début 1993, les textes fondateurs de la politique actuelle furent adoptés. Les principes de la politique de sécurité [5] reposent sur trois "piliers": l'intégration militaire et économique à l'Europe occidentale; les relations de bon voisinage; une défense nationale autonome.

En ce qui concerne le premier pilier, le document précise que la sécurité de la Hongrie sera garantie en dernière instance par l'adhésion à l'Union euro- 
péenne. En même temps, la Hongrie fera ce qu'elle peut pour entrer dans I'OTAN : avant d'avoir atteint ces objectifs, elle renforcera ses relations avec ces institutions. Le développement des relations de bon voisinage est d'ailleurs souhaité, dans la mesure où il ne nuit pas à l'intégration européenne de la Hongrie. Le second pilier sera pour l'essentiel l'occasion de renforcer la coopération dans les domaines liés aux relations économiques, aux droits de l'homme et aux droits des minorités qui sont, précisent les "principes de bases" en évoquant les minorités hongroises, "une condition nécessaire" à l'établissement des relations de bon voisinage. Le document se poursuit en rappelant la charte d'Helsinki à propos des frontières.

Le second document [6] présente les principes de la politique de défense nationale du pays. Celle-ci repose sur le triptyque coopération-dissuasiondéfense. Rien n'est dit de l'ennemi potentiel, mais on fournit une liste des facteurs pouvant mettre en cause la sécurité du pays. Celle-ci décrit d'abord les quatre types de menaces qui pèsent sur la Hongrie du fait des incertitudes régionales. Rien d'original puisque l'on évoque l'instabilité sociale et économique, les décalages, voire les contradictions, entre les divers processus de transition démocratique, les conflits interethniques liés à l'existence des minorités et aux diverses frustrations nationales, et enfin, les conflits plus directement issus de la contestation du tracé des frontières.

Le document ne se contente toutefois pas de ces généralités et la liste des menaces concrètes qui y sont évoquées rend bien compte des craintes des responsables hongrois. Dans trois hypothèses, on a envisagé l'utilisation du territoire hongrois comme par ricochet. Premièrement, dans le cas d'une guerre civile ou de conflits de cette nature se déroulant dans la région, où des groupes armés pourraient tenter d'utiliser le territoire hongrois. Deuxièmement, des groupes terroristes ou autres organisations paramilitaires pourraient aussi essayer, en dehors de toute situation de crise, soit de faire transiter armes et hommes par la Hongrie, soit de mener des actions contre ce pays. Troisièmement, en cas de conflit entraînant deux parties, l'une d'elles pourrait chercher à passer par la Hongrie pour nuire à l'autre. Est bien sûr évoquée l'évolution potentielle d'un pays voisin où des forces politiques hostiles à la Hongrie arriveraient au pouvoir et chercheraient à mettre en danger soit les intérêts économiques vitaux du pays, soit sa sécurité même en préparant une action militaire directe. Enfin, pour tenir compte des évolutions du conflit yougoslave, le document s'interroge sur les dangers induits par une participation des forces armées hongroises à des actions menées sous l'égide de l'ONU.

Tout ceci reste bien sûr au niveau de l'exercice de style, mais la diplomatie hongroise a dû réagir à deux initiatives régionales visant à changer le cadre même de la politique régionale de sécurité. Il y a d'abord eu les propositions, plus ou moins précises mais répétées, du président polonais, Lech Walesa, de créer une "OTAN bis" pour l'Europe centrale. Puis, le président ukrainien Kravtchouk a avancé l'idée d'une zone de stabilité associée à des mesures de confiance dans la région. Dans les deux cas, la diplomatie hongroise a voulu se tenir à distance, refusant de donner tout début de concrétisation à ces plans qui, selon la doctrine hongroise en vigueur, pourraient entrainer un ralentissement du processus de rapprochement avec l'Europe occidentale. 


\section{Les limites du consensus interne}

Contrairement à la politique intérieure, la politique extérieure de la Hongrie après le changement de régime a fait l'objet d'un consensus qui s'est maintenu pendant plusieurs années. Aujourd'hui le consensus n'est plus et il ne renaît de ses cendres que dans des situations de tensions, comme à l'occasion du putsch de Moscou. L'érosion du consensus s'est accélérée, de façon intéressante, non pas à l'initiative de l'opposition parlementaire, mais à cause des dissensions au sein de la majorité gouvernementale, et à propos de la question des frontières.

Dès 1991, le second parti de la coalition gouvernementale (les petits propriétaires) quitta le gouvernement, bien évidemment non sans arrière-pensées en politique intérieure, mais en formulant une politique extérieure plus nationaliste que la ligne officielle et en exigeant de nouveaux traités de paix. Le groupe parlementaire des petits propriétaires est resté majoritairement favorable au gouvernement Antall. Plus tard, en 1993, au sein même du parti dirigeant de la coalition gouvernementale (le Forum démocratique hongrois), un groupe quitta le giron antallien en refusant de ratifier le traité hungaroukrainien. C'était le premier traité de base avec un pays voisin (il n'y en a pas eu depuis) ; il incluait un article spécifiant que les deux pays renonçaient mutuellement à toute revendication territoriale à l'égard de l'autre partie. Cependant que l'opposition votait en faveur du traité, le groupe dissident du Forum votait contre et, peu après, établissait sa propre plate-forme politique avant de se transformer en parti que l'on trouve aujourd'hui à l'extrême droite de la palette politique parlementaire. Il se caractérise par un nationalisme marqué.

Le gouvernement hongrois a bien saisi le délicat problème auquel il est confronté quand il cherche à la fois à défendre les minorités et à établir de bonnes relations avec ses voisins. S'appuyant sur les traités de Paris de 1947 et sur les accords d'Helsinki, le gouve:nement rejette toute modification des frontières par la force. Ayant réaffirmé ce principe de nombreuses fois, il estime qu'il est inutile voire nuisible d'y revenir dans les accords et traités bilatéraux envisagés avec les pays voisins. Dans le cas ukrainien, il est clair que le gouvernement a passé outre à ses propres consignes, mais il n'a pas manqué de préciser qu'il s'agissait d'une exception à ne considérer en aucun cas comme ayant valeur de précédent. Il a souligné son intention de ne pas renouveler ce type de démarche dans les accords avec les autres pays voisins.

C'est d'ailleurs en refusant de s'associer à cette politique visant à éviter de traiter les frontières comme un problème, que le principal parti d'opposition (Alliance des démocrates libres) a mis fin définitivement au consensus de politique étrangère. Après des mois de réflexion, lors du débat sur les fondements de la politique de sécurité en février 1994, il se distingua des cinq autres partis parlementaires en proposant une solution à double détente. Le renoncement réciproque aux revendications territoriales entre Etats voisins pourrait être intégré à des traités bilatéraux ; mais, pour que de tels traités voient le jour, l'Alliance exige un accord sur la défense des droits des minorités.

Les programmes préparés par les partis pour les élections de mai 1994 montrent que l'époque du refus de l'ancien régime et de rupture avec l'URSS 
est bien terminée et que le consensus en politique étrangère n'est plus qu'un souvenir. Des divergences apparaîtront donc dans plusieurs domaines.

Le centre modérément nationaliste soulève de façon sélective la question des frontières tout en ne désirant pas réellement de modifications, mais en espérant pouvoir ainsi disposer d'un atout dans la négociation en vue d'améliorer le sort des minorités hongroises. La priorité accordée à l'adhésion à l'Union européenne est équivalente à celle donnée à la défense des minorités, et ce groupe pense que l'adhésion à l'OTAN se fera rapidement.

La conception libérale dominante ne situe pas au premier rang de ses priorités la question des minorités. Elle voit d'ailleurs sa solution non pas dans un cadre bilatéral mais plutôt au travers d'une solution internationale. Sa vraie priorité est l'intégration via l'Union européenne alors que l'OTAN n'est pas une priorité chronologique, le statut de membre de plein droit étant attendu pour le moment opportun, et non pas à tout prix rapidement.

La gauche modérée a à peu près la même position que le groupe précédent sur la question des frontières, mais use parfois d'une intonation plus nationale en la matière. L'adhésion à l'Union européenne est, pour elle aussi, la priorité des priorités. Elle est en revanche plus prudente quant à l'entrée dans l'OTAN. D'une part, elle souhaiterait un référendum et, d'autre part, elle insiste sur l'établissement d'un système de sécurité pour l'ensemble de la région, en lui donnant la priorité sur des démarches d'adhésion à l'OTAN pays par pays.

Avant de passer aux conclusions de cette étude, il est important de souligner que la description qui vient d'être faite est intentionnellement silencieuse sur les partis existants en tant que tels. Les axes présentés ici ne peuvent être que des références, la politique effectivement menée à l'issue des élections dépendra d'alliances que le système électoral hongrois, mélangeant proportionnelle et scrutin d'arrondissement uninominal à deux tours, rend très difficiles à prévoir, d'autant que les partis sont jeunes et assez incertains en maints domaines.

Cependant, la Hongrie a accueilli d'une tout autre manière une proposition originale, à l'origine française (plan Balladur), qui s'est transformée en projet de pacte de stabilité. Les négociations qui doivent servir de socle à cet édifice permettent d'envisager enfin après le cycle ouvert par les traités de paix mettant fin à la Première puis à la Seconde Guerre mondiale, de donner à la Hongrie un nouveau statut en Europe et de formuler un cadre satisfaisant pour les minorités nationales de l'ensemble de la région. Cela est d'autant plus important qu'une des conditions de l'entrée de notre pays dans l'Union européenne est l'établissement de relations satisfaisantes avec l'ensemble des pays voisins, de sorte que l'existence des minorités hongroises ne puisse apparaitre comme la source possible d'un conflit politique potentiellement porteur de menaces sur la sécurité de la région. La crise yougoslave a d'ailleurs incité les grandes puissances intéressées et les Etats ouest-européens à mettre l'accent sur la consolidation des frontières. Il est vrai que la Hongrie a, jusqu'à présent, cherché à donner à l'Union européenne un rôle de médiateur afin que des pays voisins, au cas où cela lui semblait nécessaire, assouplissent leurs positions quant aux droits des minorités. Avec le projet de pacte de stabilité en Europe, il semble donc possible à la diplomatie hongroise de gérer ses deux principales priorités (l'intégration européenne et la 
protection des minorités hongroises) en évitant que la question des minorités ne nuise au choix européen.

\section{Conclusion}

On peut partir d'une première constatation largement positive. La diplomatie hongroise a réussi à changer d'orientation en donnant la priorité à l'Ouest, à gagner puis à maintenir la souveraineté du pays, et à définir un cours nouveau fondé sur la définition des intérêts prioritaires de la nation. Dans ce dernier domaine, le travail n'a pas été facile puisqu'il a fallu répondre à des questions qui étaient tout simplement impensables auparavant.

Les résultats sont concrets : une avancée significative sur la voie de l'intégration européenne, un début de mise en œuvre d'une coopération régionale non plus fondée sur des cadres artificiels mais sur les besoins " naturels" des divers partenaires. De plus, la cause longtemps savamment tue des minorités hongroises à l'étranger est devenue un thème courant de débat sur la scène politique internationale.

Toutefois, deux remarques critiques s'imposent. D'une part, des contradictions sont à l'évidence apparues entre les diverses priorités de la diplomatie hongroise. D'autre part, les résultats obtenus dans les divers domaines ont été très inégaux, ce qui remet en cause la validité des hypothèses avancées pour mener telle ou telle action. Il nous faut préciser que la réflexion indépendante sur la politique étrangère n'a pas encore de tradition en Hongrie et que, par exemple, une partie des responsables s'oppose à l'examen de l'appareil de décision en politique étrangère. Le manque d'assurance en la matière n'est le privilège de personne et on peut constater, chez de nombreux acteurs non officiels intervenant sur des questions de politique étrangère, le rejet de toute réflexion sérieuse sur l'adéquation entre les buts et les moyens. En ajoutant à cela les difficultés inhérentes au renouvellement d'une bonne partie du personnel diplomatique dans une jeune démocratie où le gouvernement ne bénéficie du soutien que d'une partie des élites intellectuelles et administratives gagnées à la démocratie, il est clair que les débats sur les acquis et les faiblesses de la diplomatie du premier gouvernement vont gagner en alacrité et en intensité.

Mais la campagne électorale et les élections vont aussi amener les différentes forces politiques à comparer leurs programmes. Il est d'ores et déjà certain qu'aucune des forces qui seront en mesure soit de gouverner, soit de mener une opposition parlementaire constructive, ne veut changer fondamentalement les orientations majeures de la diplomatie hongroise. On peut toutefois prévoir un débat ravivé sur les moyens à mettre en œuvre, sur l'importance accordée à telle ou telle question. Une fois l'étape des élections passée, les partenaires européens et les voisins de la Hongrie pourront constater que ce pays reste un partenaire au comportement prévisible qui garde sur la scène internationale une attitude fondée sur sa volonté d'intégration européenne. Cela est particulièrement important car, avant la seconde législature de l'ère démocratique, la diplomatie ne manquera pas de devenir de plus en plus l'instrument de l'Etat vu comme la personnification de l'ensemble des intérêts de la nation, au delà des différences partisanes. Seule l'épreuve des élections peut offrir la démonstration que le pays a non seulement redéfini sa politique extérieure, mais que celle-ci n'est plus un simple instrument des intérêts à court terme des exécutifs successifs. 


\section{NOTES BIBLIOGRAPHIQUES}

[1] A Nemzeti Megújhodás Programja, programme de la rénovation nationale, Budapest, septembre 1990, p. 177-185.

[2] Ibid, p. 184.

[3] Országgyü lési Kézikönyv, manuel de l'Assemblée nationale, Budapest, 1992, p. 30.

[4] Félúton, A Nemzeti Megújhodás Programjänak elsó két éve, Bureau de presse du Premier ministre, Budapest, 1992, p. 19.

[5] Fact Sheets on Hungary. Basic Principles of the Security Policy of the Republic of Hungary, ministère des Affaires étrangères, Budapest, juin 1993.

[6] Voir Magyar Közlöny, 1993/48. 